\title{
The transcriptional activity of RNA polymerase $I$ is a key determinant for the level of all ribosome components
}

\author{
Arnaud Laferté, Emmanuel Favry, André Sentenac, Michel Riva, Christophe Carles, ${ }^{1}$ \\ and Stéphane Chédin \\ Laboratoire de Transcription des Gènes, Service de Biochimie et de Génétique Moléculaire, CEA/Saclay, \\ F-91191 Gif sur Yvette Cedex, France
}

\begin{abstract}
Regulation of ribosome biogenesis is a key element of cell biology, not only because ribosomes are directly required for growth, but also because ribosome production monopolizes nearly $80 \%$ of the global transcriptional activity in rapidly growing yeast cells. These observations underscore the need for a tight regulation of ribosome synthesis in response to environmental conditions. In eukaryotic cells, ribosome synthesis involves the activities of the three nuclear RNA polymerases (Pol). Although postulated, there is no clear evidence indicating whether the maintenance of an equimolar supply of ribosomal components reflects communication between the nuclear transcriptional machineries. Here, by constructing a yeast strain expressing a Pol I that remains constitutively competent for the initiation of transcription under stress conditions, we demonstrate that derepression of Pol I transcription leads to a derepression of Pol II transcription that is restricted to the genes encoding ribosomal proteins. Furthermore, we show that the level of 5S rRNA, synthesized by Pol III, is deregulated concomitantly with Pol I transcription. Altogether, these results indicate that a partial derepression of Pol I activity drives an abnormal accumulation of all ribosomal components, highlighting the critical role of the regulation of Pol I activity within the control of ribosome biogenesis.
\end{abstract}

[Keywords: Ribosome biogenesis; RNA polymerase I; transcription; yeast]

Supplemental material is available at http://www.genesdev.org.

Received March 6, 2006; revised version accepted June 1, 2006.

Biosynthesis of ribosomes monopolizes up to $80 \%$ of the transcriptional activity of rapidly growing cells through transcription of $35 \mathrm{~S}$ rDNA genes by Pol I, ribosomal protein (r-protein) genes by Pol II, and 5S rRNA synthesis by Pol III. A large body of evidence indicates that synthesis of all of these components is tightly coupled to environmental conditions, and it has become clear that the regulation of ribosome biogenesis is a determinant parameter of cell-growth control (discussed in Rudra and Warner 2004). The elucidation of the molecular mechanisms involved in this regulation would thus provide the foundation stone to our understanding of the economics of cell growth.

In yeast, ribosome biosynthesis is primarily regulated at the level of transcription (Warner 1999, 2001) and numerous data highlight the critical role of the conserved TOR (Target of Rapamycin) kinases in the expression of genes specifying ribosome components (Schmelzle and Hall 2000; Crespo and Hall 2002). Despite intensive ef-

${ }^{1}$ Corresponding author.

E-MAIL christophe.carles@cea.fr; FAX 33-1-69-08-47-12.

Article is online at http://www.genesdev.org/cgi/doi/10.1101/gad.386106. forts, the cascade of molecular events by which the inhibition of the TOR signaling pathway drives the global repression of the synthesis of ribosome components by all three transcriptional machineries remains largely unknown. Nevertheless, many studies have recently underscored the key role of transcription factors involved in the rapamycin-dependent repression of Pol I, Pol II, or Pol III for the transcription of ribosome component genes. The case of r-protein gene transcription is the most documented, and several groups have identified the interactions of the transcription factor Fhll with its coactivator Ifh 1 or its corepressor Crf1 as critical events for r-protein gene expression (Martin et al. 2004; Schawalder et al. 2004; Wade et al. 2004; Rudra et al. 2005). Other effectors have been implicated in the regulation of r-protein gene expression in response to rapamycin treatment, such as the transcription factor Sfp1 (Jorgensen et al. 2004; Marion et al. 2004), or the histone modifying factors Esal and Rpd3 (Reid et al. 2000; Rohde and Cardenas 2003). Rapamycin-dependent repression of Pol I transcription in yeast and mammalian cells targets the general transcription factor Rrn3/TIF-IA, leading to its dissociation from the rDNA promoter and its transloca- 
tion from the nucleolus to the cytoplasm (Claypool et al. 2004; Mayer et al. 2004). At variance with this proposal, phosphorylation of the Upstream Binding Factor was suggested to play a role in the TOR-dependent repression of Pol I activity in mouse cells (Hannan et al. 2003). In addition to these mechanisms that target the formation of the transcription initiation complex, it was suggested that the histone deacetylase $\mathrm{Rpd} 3$ controls the nucleolar structure and function in response to nutrient conditions (Tsang et al. 2003). Finally, the yeast Maf1 protein was identified as an essential and a specific mediator of Pol III transcriptional repression under a wide variety of stress conditions and, in particular, when cells exit the exponential growth phase (Pluta et al. 2001; Upadhya et al. 2002; Desai et al. 2005).

In cells defective for the secretory pathway or in response to nutrient starvation or rapamycin treatment, several reports have revealed a simultaneous decrease in the level of a selection of mRNAs encoding r-proteins and in the level of either the 35S rRNA (Mizuta and Warner 1994; Powers and Walter 1999) or the 5S rRNA (Li et al. 2000), or a simultaneous decrease in the levels of 35S rRNA and 5S rRNA (Clarke et al. 1996; Zaragoza et al. 1998). These observations led to the proposal that the transcription by Pol I, Pol II, and Pol III of genes specifying rRNAs or r-proteins was coregulated. No direct evidence, however, was provided to demonstrate that the concomitant deregulation of these RNAs was exerted at the transcriptional level. Thus, whether the maintenance of an equimolar supply of ribosomal components reflects the existence of communication between two, if not all three, nuclear transcriptional machineries remains an open question.

In this study, we addressed this central issue by interfering with the regulatory mechanisms of Pol I transcription and by determining the consequences of this deregulation on Pol II and Pol III transcription. We show that an attenuation of the rapamycin-dependent repression of Pol I activity leads to a concomitant attenuation of the repression of r-protein gene transcription by Pol II and to an attenuated decrease of the level of $5 \mathrm{~S}$ rRNA, synthesized by Pol III. In addition, we demonstrate that the cross-talk between the Pol I and Pol II machineries is specific and restricted to only a minor subset of class II genes, including all r-protein genes. These data are the first clear demonstration of a coupling between the synthesis of the RNA and protein moieties of the eukaryotic ribosome. Altogether, our results are consistent with a working model in which Pol I activity plays a determinant role within the control of the level of ribosome biogenesis.

\section{Results}

The dissociation of the Pol I-Rrn3 complex mediates the rapamycin-dependent repression of Pol I

Pol I has the sole essential purpose of transcribing the ribosomal RNA precursor gene (Nogi et al. 1991). In Saccharomyces cerevisiae, four general transcription factors are specifically required for initiation of Pol I transcription (Nomura 2001; Grummt 2003): the multimeric complexes UAF and CF, which bind the rDNA promoter in association with TBP, and the monomeric transcription factor Rrn3, which interacts with Pol I to form the Pol I-Rrn3 complex. Both in yeast and mammals, the initiation-competent subpopulation of Pol I is the Pol I-Rrn3 complex (Milkereit and Tschochner 1998; Miller et al. 2001; Nomura 2001; Grummt 2003). The formation and/or stability of this complex likely constitutes a key regulatory switch of Pol I transcription (Brun et al. 1994; Milkereit and Tschochner 1998). In particular, downregulation of rRNA synthesis during stationary phase of growth correlates with a lower recovery of Pol I-Rrn3 complexes (Milkereit and Tschochner 1998).

To interfere in vivo with the mechanisms controlling Pol I activity via the level of Pol I-Rrn3 complex, we constructed a yeast strain expressing a nondissociable complex. The two essential genes encoding Rrn3 and A43, the Pol I subunit interacting with Rrn3 (Peyroche et al. 2000), were inactivated and complemented by a gene encoding the Rrn3-A43 fusion protein (see Materials and Methods). This strain, named CARA (for Constitutive Association of Rrn3 and A 43 ), was viable and displayed the same doubling time as a wild-type strain (Supplemental Fig. S1).

Pol I purified from CARA cells lacked the A43 polypeptide as expected, but otherwise displayed a canonical subunit composition and contained an additional polypeptide of $120 \mathrm{kDa}$ that matched the estimated molecular weight of the fusion protein. This $120-\mathrm{kDa}$ polypeptide was recognized by anti-Pol I (Fig. 1A), by antiRrn3 (Supplemental Fig. S2), and by anti-A43 antibodies, which generated a signal of similar intensity with the endogenous A43 subunit in the wild-type Pol I when identical amounts of enzymes were analyzed (Fig. 1A). These data indicated that the intact Rrn3-A43 fusion protein was stably and stoichiometrically assembled within the enzyme. In view of the proposed regulatory role of the Pol I-Rrn3 complex, we investigated the response of CARA cells to rapamycin. This inhibitor of TOR kinases, which mimics nutrient-starvation conditions (Crespo and Hall 2002), leads to Pol I and Pol III transcriptional repression (Zaragoza et al. 1998; Powers and Walter 1999) and to a drastic modification of the mRNAs expression profile with a global decrease of Pol II transcription (Shamji et al. 2000; Preiss et al. 2003). In vitro Pol I-specific transcription was carried out using partially purified extracts (PA600) prepared from wildtype or CARA cells treated or not with rapamycin and containing similar amounts of Pol I (Supplemental Fig. S3). Similar levels of in vitro Pol I-specific transcriptional activity were detected in extracts from untreated wild-type or CARA cells (Fig. 1B, lanes 1,2). Remarkably, while extracts from rapamycin-treated wild-type cells lost most rDNA transcriptional activity as previously reported (Fig. 1B, lane 3; Hannan et al. 2003; Mayer et al. 2004), extracts from rapamycin-treated CARA cells retained the same level of activity as untreated wild-type or CARA cell extracts (Fig. 1B, lane 4). The inactive ex- 
Laferté et al.

A

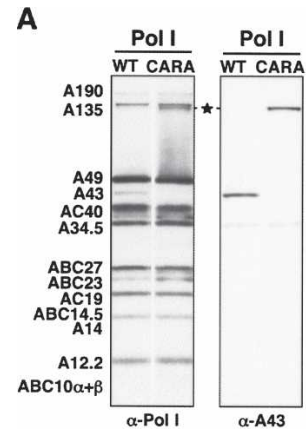

B

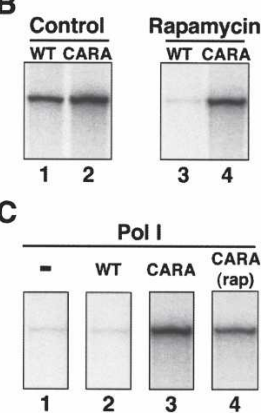

Figure 1. Pol I from CARA cells mimics a nondissociable Pol I-Rrn3 complex. (A) Western blot analysis of purified Pol I. The subunit composition of the same amount of Pol I purified from wild-type (WT) or CARA cells grown in rich medium to mid-log phase was analyzed by Western blot using anti-Pol I or anti-A43 antibodies. Both forms of enzyme display the same subunit composition, except that the A43 protein is missing in the Pol I purified from CARA cells and is replaced by the Rrn3-A43 fusion protein $(\star)$, which is stably and stoichiometrically assembled. $(B)$ Repression of in vitro Pol I transcription by rapamycin. WT or CARA cells were grown in rich medium to mid$\log$ phase, treated with rapamycin for $30 \mathrm{~min}$ (Rapamycin) or not (Control), and then harvested at the same optical density $\left(\mathrm{OD}_{600}=1.5\right)$. Partially purified extracts $(\mathrm{PA} 600)$ from WT (lanes 1,3 ) or CARA cells (lanes 2,4) containing the same amount of Pol I (see Supplemental Fig. S3), were assayed for in vitro Pol I-specific transcription using a mini-rDNA gene as template. Without rapamycin treatment, efficient Pol I transcription is observed in both WT and CARA extracts (lanes 1,2). When cells were treated with rapamycin, traces of Pol I transcription were detected in WT extract (lane 3), whereas CARA extract remained fully active (lane 4). (C) Reactivation of rapamycintreated WT cell extract. Extracts prepared from rapamycintreated WT cells, deficient for Pol I transcription (lane 1) were complemented by addition of $200 \mathrm{ng}$ of Pol I purified either from untreated WT cells (lane 2), untreated CARA cells (lane 3), or rapamycin-treated CARA cells (lane 4). Enzyme prepared from untreated and rapamycin-treated CARA cells reactivates Pol I transcription of rapamycin-treated WT cell extract, whereas Pol I prepared from WT cells has no effect.

tract from rapamycin-treated wild-type cells was fully reactivated by adding-back Pol I purified from untreated CARA cells (Fig. 1C, lanes 1,3) or from rapamycintreated CARA cells (Fig. 1C, lane 4). In contrast, a preparation of purified Pol I isolated from untreated wild-type cells, which does not contain any detectable amount of Rrn3 (Supplemental Fig. S2), was not able to reactivate the extract from rapamycin-treated wild-type cells (Fig. 1C, lane 2). Addition of purified recombinant Rrn3 had no impact on reactivation by wild-type or CARA Pol I of the inactive, rapamycin-treated wild-type extracts (Supplemental Fig. S4). These results demonstrate that Pol I purified from the CARA strain mimics an active, nondissociable Pol I-Rrn3 complex resistant to rapamycin treatment. In addition, these data confirm that the rapamycin-dependent repression of Pol I targets Rrn3 (Claypool et al. 2004; Mayer et al. 2004) and demonstrate that the Pol I-Rrn3 interaction is the major target of TOR-dependent regulation of rDNA transcription, rein-

forcing recent observations reported in mouse cells during oxidative or ribotoxic stress (Mayer et al. 2005).

\section{The abundance of ribosome components is dependent on Pol I transcriptional activity}

Next, we investigated the in vivo effect of rapamycin treatment on Pol I transcription. The decrease of the 35S rRNA level was considerably delayed in CARA cells compared with wild-type cells as monitored by primer extension analysis (Fig. 2A). In contrast, repression of the control gene encoding actin was similar in both strains

A

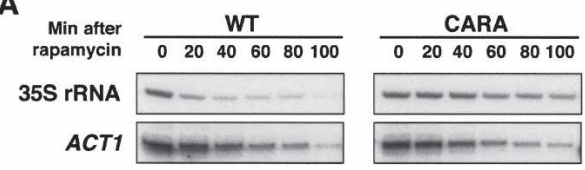

B
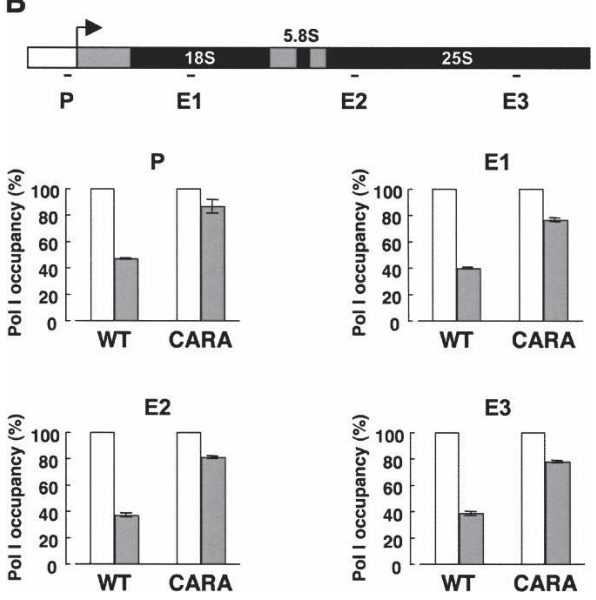

Figure 2. In vivo deregulation of Pol I transcription in CARA cells. (A) Primer extension analyses of $35 \mathrm{~S}$ rRNA and ACT1 mRNA. Wild-type (WT) or CARA cells were grown in rich medium to mid-log phase and rapamycin was added $(t=0 \mathrm{~min})$. At the indicated times, the same number of cells were harvested, total RNAs were extracted, and the amount of $35 \mathrm{~S}$ rRNA and ACT1 mRNA was determined by primer extension analysis. The decrease of the $35 \mathrm{~S}$ rRNA level is attenuated in CARA cells compared with WT cells (see quantification in Supplemental Fig. S6), whereas the ACT1 mRNA level is identical in the two strains. (B) ChIP analysis of the occupancy of the $35 \mathrm{~S}$ rDNA by Pol I. Chromatin extracts were prepared from the same number of WT or CARA cells grown in rich medium to mid-log phase either in the absence of rapamycin (white histograms) or after a 20-min rapamycin treatment (gray histograms). ChIP experiment was performed using anti-A190 antibodies. Quantification was performed by Real-Time PCR, and the occupancy of the $35 \mathrm{~S}$ rDNA by Pol I at the promoter region $(P)$ or along the transcribed region $(\mathrm{E} 1[+1430,+1536]$, E2 $[+3551,+3657]$, and E3 $[+5648,+5740]$ as indicated) after the rapamycin treatment is represented as a percentage of the occupancy without rapamycin. Standard deviation is calculated from two independent experiments. During the rapamycin treatment, the decrease of Pol I occupancy of the 35S rDNA is attenuated in CARA cells compared with WT cells. 
(Fig. 2A), suggesting that the modification of the $35 \mathrm{~S}$ rRNA level in CARA cells does not reflect a global deregulation of nuclear transcription. Importantly, the level of $35 \mathrm{~S}$ rRNA monitored by primer extension analysis is a measurement of the equilibrium between rDNA transcription and pre-rRNA processing. Because of the strategy used to generate the CARA strain, it was likely that the delay observed in CARA cells reflected a deregulation of Pol I transcription, even if we could not formally exclude that an impaired processing of the $35 \mathrm{~S}$ rRNA may have also been involved. To confirm that the deregulation of the 35S rRNA level in CARA cells in the presence of rapamycin occurred at the transcriptional level, we monitored the occupancy of the 35S rDNA by Pol I using chromatin immunoprecipitation (ChIP) experiments. As expected, the decrease in the level of Pol I on the $35 \mathrm{~S}$ rDNA was significantly attenuated in CARA cells compared with wild-type cells following rapamycin treatment (Fig. 2B), whereas Pol II recruitment on the promoter of the control gene ACT1 was similar in both strains (see Fig. 5D, below). Note that in CARA cells this attenuation was observed up to $100 \mathrm{~min}$ of rapamycin treatment (Supplemental Fig. S5). Altogether, these data indicate that upon rapamycin treatment, the transcription of the 35S rDNA in CARA cells is deregulated.

To reinforce this conclusion, we performed in vivo $\left[{ }^{3} \mathrm{H}\right]$-uracil RNA pulse-labeling experiments. The level of class I mature rRNAs (25S, 18S, and 5.8S) neosynthesized in the presence of rapamycin was significantly higher in CARA cells than in wild-type cells (Fig. 3A). Uracil uptake between the two strains being similar (data not shown), we concluded that the in vivo repression of Pol I transcription was markedly attenuated in the CARA strain in the presence of rapamycin.

Unexpectedly, the in vivo $\left[{ }^{3} \mathrm{H}\right]$-uracil RNA pulse-labeling experiments revealed that the level of 5S rRNA was deregulated in the CARA strain concomitantly with the deregulation of rRNA synthesis by Pol I (Fig. 3A), suggesting that the rapamycin-dependent decrease in the level of 5S rRNA was correlated to the control of Pol I activity. To determine whether Pol I activity directly impacted Pol III transcription of the 5S rDNA, we monitored the occupancy of the $5 \mathrm{~S}$ rDNA promoter by Pol III using a ChIP assay. Upon rapamycin treatment (Fig. 3B, gray histograms), the level of Pol III on the 5S rDNA was significantly lower in both wild-type and CARA cells compared with the nontreated cells. This result suggested that 5S rDNA transcription by Pol III was repressed in vivo in the presence of rapamycin, as demonstated in vitro in cell free extracts (Zaragoza et al. 1998). Importantly, the extent of this transcriptional repression, as judged by the level of occupancy of the gene by Pol III, was not significantly different between wild-type and CARA cells (Fig. 3B). These data suggested that the attenuation of the decrease of the level of 5S rRNA monitored by RNA pulse labeling in CARA cells during rapamycin treatment did not reflect an attenuation of the repression of 5S rDNA transcription.

The above results showed that, upon rapamycin treatment, the level of all rRNAs was prolonged in cells in
A
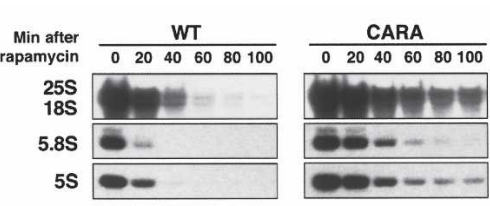

B

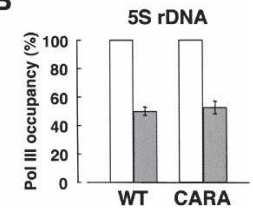

Figure 3. Attenuated repression of Pol I transcription triggers deregulation of the $5 S$ rRNA level. (A) In vivo labeling of mature rRNAs. Wild-type (WT) or CARA cells were grown in rich medium to mid-log phase before the addition of rapamycin $(t=0$ $\mathrm{min})$. At the indicated times, the same number of cells were collected and incubated with $150 \mu \mathrm{Ci}$ of $\left[{ }^{3} \mathrm{H}\right]$-uracil for $20 \mathrm{~min}$. Total RNAs were extracted and the same amount $\left(3 \mu g_{\text {; }}\right.$ see Supplemental Fig. S7) was analyzed by gel electrophoresis under denaturating conditions prior to autoradiography. Attenuated repression of the synthesis of 25S, 18S, and 5.8S rRNAs by Pol I and of the level of 5S rRNA, synthesized by Pol III, is observed in CARA cells (see quantification in Supplemental Fig. S7). (B) ChIP analysis of the occupancy of the 5S rDNA by Pol III. Chromatin extracts were prepared from the same number of WT or CARA cells containing a HA-tagged C160 Pol III subunit (see Materials and Methods). Cells were grown in rich medium to mid-log phase either in the absence of rapamycin (white histograms) or after a 20-min rapamycin treatment (gray histograms). ChIP experiment was performed using anti-HA antibodies. Quantification was performed by Real-Time PCR, and the occupancy of the $5 \mathrm{~S}$ rDNA by Pol III after the rapamycin treatment is represented as a percentage of the occupancy without rapamycin. Standard deviation is calculated from five independent experiments. During the rapamycin treatment, the decrease of Pol III occupancy onto the 5S rDNA is not significantly different between WT and CARA cells.

which repression of Pol I transcription was specifically attenuated. This observation prompted us to determine whether the partial deregulation of Pol I activity during rapamycin treatment also impacted the level of particular Pol II transcripts. To address this question, we compared the global Pol II activity in the wild-type and CARA strain upon rapamycin treatment using genomewide analysis. Total RNAs from rapidly growing wildtype and CARA cells were extracted, labeled with Cy5 and $\mathrm{Cy} 3$, respectively, and used to probe a microarray harboring all yeast ORFs. Without rapamycin, the mRNA expression profiles of the two strains were indistinguishable (Fig. 4). In particular, the level of mRNAs encoding r-proteins (black spots) was identical in wildtype and CARA cells. This observation was consistent with their identical doubling times during the exponential phase of growth. Upon rapamycin treatment, we observed that almost all mRNAs (97\%) were similarly regulated in both strains, except for 147 mRNAs (i.e., $2.5 \%$ of the mRNAs analyzed), which were significantly and specifically over-represented in CARA cells (Fig. 4; Supplemental Table S1). Spot per spot analysis revealed that 128 of those 147 mRNAs species encoded r-proteins. For these 128 transcripts, the average ratio of abundance in CARA versus wild-type cells was 7.7 in the presence of rapamycin (1.0 without rapamycin). Over the 138 genes specifying r-proteins in the genome of $S$. cerevisiae, 131 were represented on the microarray, indicating 
Laferté et al.
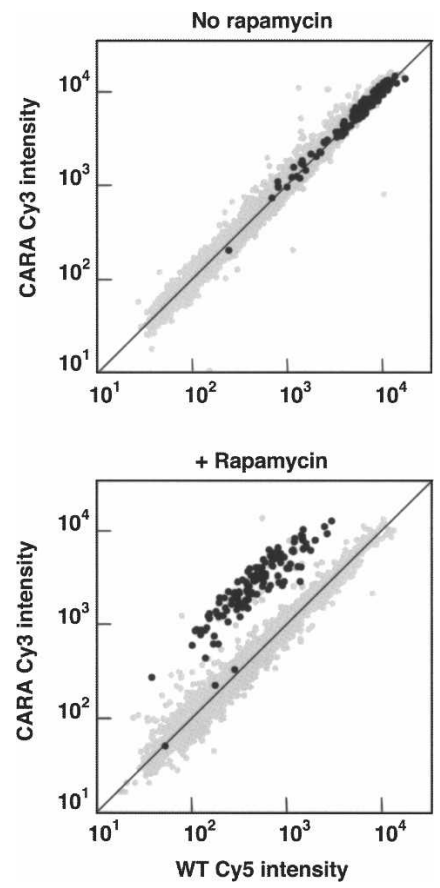

Figure 4. Attenuation of rapamycin-dependent Pol I transcription repression impairs specifically the level of mRNAs encoding r-proteins. Genome-wide analysis of expression levels of class II genes. Wild-type (WT) or CARA cells were grown in rich medium to $\mathrm{OD}_{600}=1$ (mid-log phase) and further incubated for 60 min with rapamycin ("+ Rapamycin") or without rapamycin ("No rapamycin"). Cells were harvested and total RNAs were extracted. RNAs $(20 \mu \mathrm{g})$ were labeled by reverse transcription in the presence of Cy5 dUTP (WT) or Cy3 dUTP (CARA) (see Materials and Methods) and used to probe a microarray harboring all yeast ORFs. Results were analyzed using the GeneSpring software (Silicon Genetics). A scatterplot representation of expression levels is displayed. Each individual spot corresponds to a gene, and its location on the diagonal indicates that the abundance of the corresponding mRNA is similar in both strains. mRNAs encoding r-protein genes (black spots) are present at the same level in untreated WT and CARA cells but are specifically overrepresented in CARA cells in the presence of rapamycin (from a threefold to a 13 -fold factor, with an average factor of 7.7).

that the level of all mRNAs encoding r-proteins is specifically deregulated in CARA cells during rapamycin treatment. Note that the level of total RNAs extracted from the same number of wild-type and CARA cells in the presence of rapamycin was about half of that extracted when cells were untreated. However, for each condition (i.e., without or with rapamycin), the amounts of total RNAs recovered from CARA or wild-type cells were identical.

The specific overrepresentation of mRNAs encoding r-proteins in CARA cells during the rapamycin treatment was confirmed by Northern blot (Fig. 5A) and primer extension analyses (Fig. 5B). In the presence of rapamycin, the decrease in the level of mRNAs encoding r-proteins was strongly attenuated in CARA cells, whereas down-regulation of the control mRNA encoding actin was similar in both strains. To determine whether this specific deregulation of the level of mRNAs encoding r-proteins in CARA cells was exerted at the transcriptional level, we monitored the occupancy of the promoter of r-protein genes by Pol II using ChIP experiments. As shown in Figure 5C, upon rapamycin treatment (gray histograms), the recruitment of Pol II to r-protein gene loci was significantly higher in CARA cells than in wild-type cells. In contrast, Pol II recruitment on the control genes (ACT1 and $A D H 1)$ was undistinguishable between the two strains (Fig. 5D). Altogether, the above data indicated that, during rapamycin treatment, the repression of r-protein gene expression by Pol II was reduced in cells where repression of Pol I transcription was specifically attenuated.

The transcriptional activity of Pol I is a key parameter for the level of ribosomes

We next wondered whether, during rapamycin treatment, the relative accumulation of $35 \mathrm{~S}$ rRNA, 5S rRNA, and mRNAs encoding r-proteins in CARA cells led to an increased number of assembled ribosomes compared with wild-type cells. To address this issue, we compared the amount of ribosomes present in the same number of wild-type or CARA cells during rapamycin treatment by estimating the amount of $40 \mathrm{~S}$ and $60 \mathrm{~S}$ ribosomal particles after dissociation of polysomes and $80 \mathrm{~S}$ particles. As depicted in Figure 6, the amount of $40 \mathrm{~S}$ and $60 \mathrm{~S}$ ribosomal particles was nearly identical in the two strains before rapamycin treatment and decreased gradually in the presence of the drug but to a lower extent in CARA cells. After $180 \mathrm{~min}$ of rapamycin treatment, CARA cells contained twice the amount of 40S and 60S observed in wild-type cells, indicating that the partial derepression of all classes of genes specifying ribosomal components in CARA cells led to an attenuated decrease of the amount of assembled ribosomal particles. This observation suggests that in the presence of rapamycin, the relative excess of ribosomal components in CARA versus wild-type cells is indeed properly assembled into ribosomal particles. In addition, when ribosome analysis was performed under nondissociating conditions, we did not observe the presence of half-mers during rapamycin treatment (data not shown), suggesting an equimolar production of $40 \mathrm{~S}$ and $60 \mathrm{~S}$ particles in wild-type and CARA cells.

The critical impact of Pol I activity on the level of ribosome components is not restricted to rapamycin treatment

Next, we investigated whether the influence of Pol I activity on the other two transcriptional machineries was restricted to rapamycin treatment or whether it was a general feature of the transcriptional control of genes encoding ribosome components. Because the stability of the Pol I-Rrn3 complex was suggested to be a key parameter for Pol I repression during stationary growth 
A

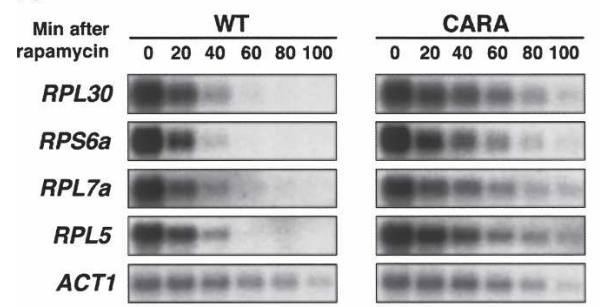

B

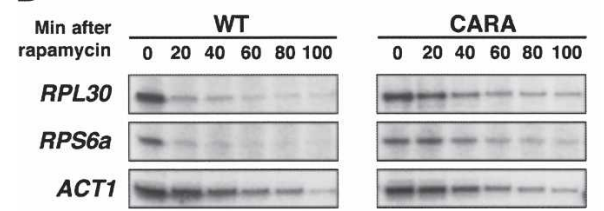

C

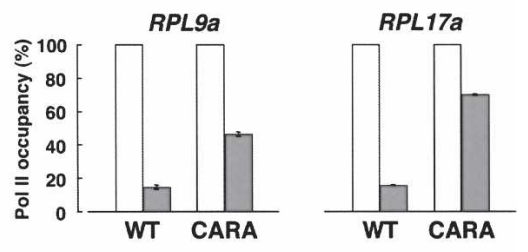

D

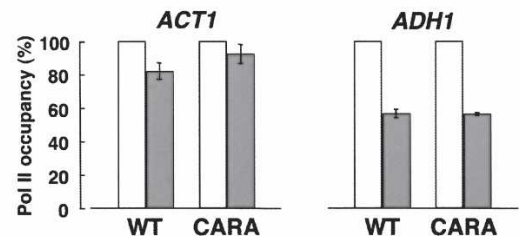

Figure 5. The deregulation of mRNAs encoding r-proteins in CARA cells during rapamycin treatment is exerted at the transcriptional level. $(A, B)$ Northern blot and primer extension analyses. Wild-type (WT) or CARA cells were grown in rich medium to mid-log phase before rapamycin treatment $(t=0$ $\mathrm{min})$. At the indicated times, the same number of cells was harvested and total RNAs were extracted. (A) Northern blot analyses were performed using probes hybridizing to four r-protein mRNAs and to the control ACT1 mRNA, as indicated. $(B)$ Using primer extension analyses, the amount of two r-protein mRNAs was determined, and the ACT1 mRNA was used as a control. For both experiments, the decrease in the level of mRNAs encoding r-proteins is attenuated in CARA cells compared with WT cells, while the level of the control ACT1 mRNA is similarly down-regulated in the two strains (see quantification in Supplemental Fig. S8A,B). $(C, D)$ ChIP analysis of the occupancy of RPL9a, RPL17a $(C)$ and $A C T 1, A D H 1(D)$ promoters by Pol II. Chromatin extracts were prepared from the same number of WT or CARA cells grown in rich medium to mid-log phase either in the absence of rapamycin (white histograms) or after a 20-min treatment (gray histograms). ChIP experiments were performed using anti-CTD antibodies. Quantification was performed by Real-Time PCR, and the occupancy of the different promoters by Pol II is represented as a percentage of the occupancy without rapamycin. Standard deviation is calculated from three independent experiments. During rapamycin treatment, the level of Pol II on the promoters of r-protein genes is higher in CARA cells than in WT cells, whereas the level of Pol II on the promoter of the two control genes ACT1 and ADH1 is identical in the two strains. phase (Milkereit and Tschochner 1998; Grummt 2003; Mayer et al. 2004), we might infer from our data obtained during rapamycin treatment that an attenuation of the repression of ribosome-component synthesis should be observed as CARA cells enter post-diauxic growth phase. To test this hypothesis, we harvested wild-type and CARA cells either in mid-log phase or at the same stage of diauxic growth transition (i.e., at the same $\mathrm{OD}_{600}$, the growth curves of wild-type and CARA culture being identical) (Supplemental Fig. S1). Pol I activity was assessed by an in vitro transcription assay, primer extension analysis, and in vivo RNA pulse labeling, whereas Pol III transcriptional activity was estimated by in vivo RNA pulse labeling. As expected, we observed that the repression of Pol I activity was attenuated in CARA cells compared with wild-type cells and that the level of 5S rRNA synthesized by Pol III was deregulated to the same extent (data not shown). Pol II activity was evaluated by microarray analysis. As reported above, the distribution of expression ratios for all mRNAs between rapidly growing wild-type and CARA cells, including those of r-proteins, was identical (Fig. 7, mid-log phase). During the diauxic transition, this distribution was unchanged for the vast majority of mRNAs. In contrast, the genes encoding r-proteins were significantly more expressed in CARA cells compared with wild-type cells (Fig. 7, diauxic transition), suggesting that the relation between the level of r-protein gene transcription and Pol I activity, which was revealed under rapamycin treatment, was also relevant under physiological conditions.

\section{Discussion}

Here, we demonstrate that the regulation of Pol I activity is a key player for the control of the level of all ribosome

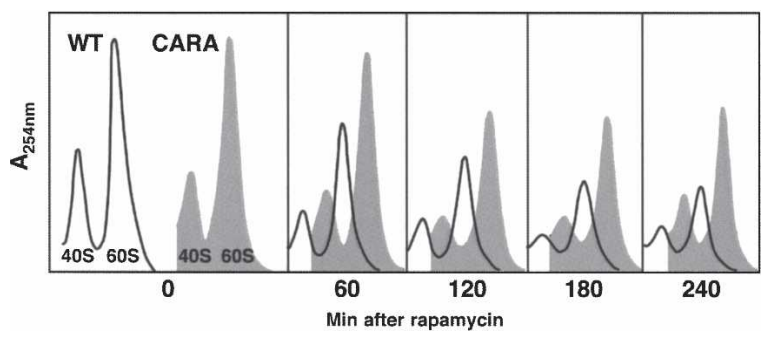

Figure 6. The excess of ribosomal components in CARA vs. wild-type cells is assembled into ribosomes during rapamycin treatment. Quantification of $40 \mathrm{~S}$ and 60S ribosomal particles. Rapamycin was added to a mid-log phase culture of wild-type (WT) or CARA cells grown in rich medium. At the indicated times, $10^{9}$ cells were withdrawn, resuspended in a buffer without $\mathrm{MgCl}_{2}$ (see Materials and Methods), and disrupted by vigorous vortexing in the presence of glass beads. After clarification, the lysate was loaded onto a $5 \%-35 \%$ linear gradient of sucrose, and $40 \mathrm{~S}$ and $60 \mathrm{~S}$ ribosomal particles were separated by centrifugation under dissociating conditions. Fractions were collected from the bottom, and the amount of $40 \mathrm{~S}$ and $60 \mathrm{~S}$ ribosomal particles was determined by measuring the absorbance at $254 \mathrm{~nm}$. During rapamycin treatment, the amount of ribosomal particles decrease in WT and CARA cells, but this reduction is strongly attenuated in CARA cells. 

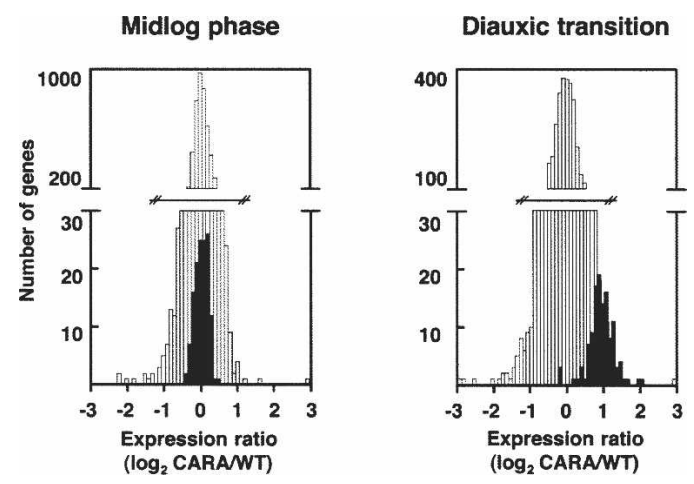

Figure 7. Pol I activity impacts the transcriptional regulation of r-protein genes during diauxic growth transition. Genomewide analysis of expression levels of class II genes. Wild-type (WT) or CARA cells were grown in rich medium either to mid$\log$ phase or $90 \mathrm{~min}$ after the end of the log phase at the diauxic transition (as indicated in Supplemental Fig. S1). After total RNA extraction, $20 \mu \mathrm{g}$ of RNAs were labeled by reverse transcription in the presence of Cy5 dUTP (WT) or Cy3 dUTP (CARA) (see Materials and Methods) and used to probe a microarray harboring all yeast ORFs. Results were analyzed using the GeneSpring software (Silicon Genetics). For all mRNAs, the ratio of expression in CARA cells over WT cells (CARA/WT) was calculated. These values were transformed to a $\log 2$ scale, and the resulting distribution of ratios is depicted (r-protein genes, black histograms; non-r-protein genes, white histograms). During the mid-log phase, the distribution of expression ratios for r-protein genes is centered on zero, indicating that these genes are similarly expressed in both strains. During diauxic transition, mRNAs encoding r-protein are significantly overrepresented in CARA cells compared with WT cells.

components. Numerous studies have previously investigated the transcriptional repression of genes specifying ribosome components ( $\mathrm{r}$-protein genes, 35S rDNA, and $5 \mathrm{~S}$ rDNA) in response to different stresses. As mentioned in the introduction, these studies have reported a concomitant down-regulation of the transcription of a selection of r-protein genes with either 35S rDNA or 5S rDNA or 35S rDNA concomitantly with 5S rDNA. Such observations of a concomitant repression led to the proposal that these genes are subject to coordinated regulation. Indeed, if a set of genes is coregulated, these genes should be concomitantly down-regulated under repressive conditions. However, the reverse does not necessarily hold true. At least two important criteria should be fulfilled to firmly conclude that two genes are coregulated when they are concomitantly down-regulated under given repressive conditions. First, this down-regulation should be selective, i.e., restricted to a limited number of genes. This requires estimating the regulation of a large number of genes, if not all. Second, it should be observed in cells whose physiological state is as close as possible to that of wild-type cells (i.e., displaying comparable doubling times). These two criteria were not met in the previous studies. Here, we attenuated in vivo the rapamycindependent repression of the Pol I transcription (CARA strain) and investigated the impact of this deregulation on the level of class II and class III RNAs. Notably, in the absence of stress, the CARA cells display a wild-type phenotype. During rapamycin treatment, we observed a concomitant deregulation of the level of the 5S rRNA synthesized by Pol III as well as an attenuation of repression of r-protein gene transcription by Pol II. Importantly, the concomitant deregulation of Pol II transcription was selectively restricted to a very small subset of class II genes, including all r-protein genes and 19 additional class II genes (nine specifying proteins involved in ribosome biogenesis or translation, four encoding proteins of unrelated function, and six corresponding to hypothetical ORFs) (see Supplemental Table S1). These genes, however, are not systematically deregulated in CARA cells under all stress conditions /diauxic transition and tunicamycin treatment, for example; data not shown), contrary to r-protein genes. Altogether, these results demonstrate unambiguously for the first time a coregulation between the Pol I and Pol II transcriptional machineries with respect to the synthesis of ribosome components. Other studies on ribosome assembly have demonstrated the coordinated regulation at the transcriptional level of $>100$ genes encoding proteins other than r-proteins implicated in rRNA processing and ribosome assembly, which form the Ribi regulon (Jorgensen et al. 2004). Remarkably, none of the Ribi regulon genes was specifically deregulated in CARA cells during rapamycin treatment, demonstrating that the cross-talk between the Pol I and the Pol II systems is limited to the synthesis of ribosome components.

Another striking observation made in this study is the higher level of 5S rRNA in CARA cells compared with wild-type cells during rapamycin treatment, as judged by in vivo $\left[{ }^{3} \mathrm{H}\right]$-uracil RNA pulse-labeling experiments. In the particular case of the 5S rRNA, the amount of labeled RNA is commonly thought to directly reflect the level of Pol III transcription of the 5S rDNA. Therefore, it is tempting to conclude that during rapamycin treatment the repression of $5 \mathrm{~S}$ rDNA transcription by Pol III was attenuated in cells where repression of Pol I transcription was specifically relieved. Pol III occupancy of the $5 \mathrm{~S}$ rDNA gene, monitored by ChIP experiments, does not, however, support this hypothesis. Upon rapamycin treatment, the decrease in enzyme occupancy of the $5 \mathrm{~S}$ rDNA observed in both CARA and wild-type cells corroborates studies demonstrating a rapamycin-dependent repression of class III gene transcription by Pol III (Upadhya et al. 2002; Oficjalska et al. 2006) and confirms, in particular, in vitro data showing the repression of the 5S rDNA transcription under similar conditions (Zaragoza et al. 1998). Surprisingly enough, we did not evidence any significant difference in 5S rDNA occupancy by Pol III between CARA and wild-type cells in the presence of rapamycin. Because recent ChIP analyses highlighted a correlation between the level of Pol III occupancy of a gene and its level of expression under different stress conditions (Harismendy et al. 2003; Roberts et al. 2003; J. Soutourina, pers. comm.), our data suggest that during rapamycin treatment the attenuated decrease of the level of the 5S rRNA in CARA cells does not result from an attenuation of the transcriptional re- 
pression of the 5S rDNA. Because RNA levels estimated by in vivo $\left[{ }^{3} \mathrm{H}\right]$-uracil RNA pulse-labeling experiments result from an equilibrium between synthesis, processing, and degradation, we propose that, under rapamycin treatment, the attenuated decrease of the level of the $5 \mathrm{~S}$ rRNA in CARA cells may reflect its stabilization by the excess of r-protein able to interact with this RNA species (Ulbrich et al. 1980; Deshmukh et al. 1993).

In conclusion, our results demonstrate that, during rapamycin treatment, the repression of r-protein gene expression by Pol II and the decrease of the level of $5 \mathrm{~S}$ rRNA were attenuated in cells where repression of Pol I transcription was specifically attenuated. We evidence the existence of a cross-talk between the Pol I and the Pol II transcriptional machineries that is restricted to the synthesis of ribosome components. While Pol III transcription does not appear to be coregulated with Pol I activity, the amount of 5S rRNA is concomitantly deregulated with that of the $35 \mathrm{~S}$ rRNA.

Our data support the idea that the regulation of Pol I activity plays an important role within the control of the supply of ribosomes components. This suggestion is reminiscent of the situation observed in Escherichia coli, where the control of ribosome synthesis depends on the control of rRNA transcription (Gourse et al. 1996). Although additional mechanisms linking Pol I activity to the processing and/or stability of class II and class III RNAs may exist (Briand et al. 2001), the concept that the synthesis of r-protein mRNAs by Pol II and the level of 5S rRNA depend, at least under some conditions, on the level of Pol I activity is consistent with several former observations: (1) Under certain environmental conditions, rRNA transcription can occur in the absence of r-protein synthesis (Warner 1999); (2) conditional inactivation of Pol II machinery does not appear to affect directly Pol I and Pol III activities (Cormack and Struhl 1992); and (3) derepression of Pol III transcription has no impact on Pol I activity and r-protein gene transcription (Upadhya et al. 2002). In apparent contrast with this proposal, Pol II transcription was found to be uncoupled in mutant cells with defective Pol I assembly (Wittekind et al. 1990). More recently, it was observed that cells were able to adjust their transcription of the $35 \mathrm{~S}$ rDNA to a reduced production of r-proteins in a $\Delta f h l 1$ context (Rudra et al. 2005). However, in these cases, the data were obtained in mutant cells whose physiology (i.e., the cell growth rate) was severely and deleteriously affected. The concerted regulation of the transcriptional systems for the supply of the ribosome components demonstrated in our work does not display such limitations. Indeed, in the absence of a repressive stimulus (i.e., without rapamycin or during the exponential phase of growth), CARA cells display a wild-type phenotype (as evidenced by their doubling time, mRNA expression pattern, and the amount of assembled ribosomal particles identical to those of isogenic wild-type cells), allowing the characterization of physiological responses of the nuclear transcriptional machineries under relevant regulatory pressure.

The possibility that Pol I activity is a determinant for the level of ribosome components raises the important question of how the cell senses the level of Pol I activity and how this information is transmitted to Pol II to adjust the level of mRNAs encoding r-proteins. Schematically, at least two generic types of mechanisms can be envisaged. Firstly, the cell could sense 35 rRNA levels directly (for example, by titrating parts of the transcript that are stoichiometrically produced during the processing of the primary transcript) or indirectly (by sensing the number of activated rDNA promoters or the amount of activated Pol I molecules). Secondly, given that the nucleolus appears as a steady-state compartment directly linked to Pol I transcriptional activity (Mayer and Grummt 2005), one might hypothesize that the formation and/or the maintenance of this nuclear territory influences transcription of nearby genes. In this respect, it is tempting to speculate that the genes encoding ribosomal proteins might be close or linked in some way to the nucleolus and that their transcriptional activity might depend on the integrity of the nucleolar structure, paralleling the situation reported for other class II genes whose transcriptional state was shown to be dependent on their nuclear localization (Dernburg et al. 1996; Gerasimova et al. 2000; Feuerbach et al. 2002). The verification or otherwise of these hypothesis would require exhaustive studies that should, in turn, be invaluable in deciphering the complex network of molecular mechanisms controlling the production of ribosome components.

\section{Materials and methods}

Plasmids, strains, and medium

$R R N 3$ and RPA43 open reading frames were fused using a twostep PCR strategy. Briefly, RRN3 was amplified by PCR with the SC46 primer introducing a BamHI restriction site at the 5' end of RRN3 and the SC47 primer replacing the Stop codon of $R R N 3$ by a HindIII restriction site and introducing $21 \mathrm{bp}$ of the 5' sequence of RPA43. RPA43 was amplified by PCR with the SC48 primer introducing 21 bp of the $3^{\prime}$ sequence of $R R N 3$ (the Stop codon being replaced by a HindIII restriction site) at the $5^{\prime}$ end of RPA43 and the SC49 primer introducing a EcoRI restriction site at the $3^{\prime}$ end of RPA43.

\section{SC46: 5'-TCAGCGGGATCCATGATGGCTTTTGAGAATA CAAG-3' \\ SC47: 5'-GGCTCTTTTTACTTGTGACATAAGCTTGTCAT CCGACCCATCACTTTC-3' \\ SC48: 5'-GAAAGTGATGGGTCGGATGACAAGCTTATGT \\ CACAAGTAAAAAGAGCC-3' \\ SC49: 5'-TCAGCGGAATTCCTAATCACTATCACTCGATT CACC- $3^{\prime}$}

The two PCR products were mixed and used as a template for a second-step PCR using SC46 and SC49 as primers. The final PCR product was cloned between the BamHI and EcoRI restriction sites of the yeast expression vector pGEN $(2 \mu, T R P 1, P G K 1$ promoter) to give the pGEN-RRN3-RPA43 plasmid. CARA strain was obtained by transforming the YPH500 strain (Sikorski and Hieter 1989) with the pGEN-RRN3-RPA43 plasmid and disrupting the endogenous RRN3 $\left(\Delta r r n 3:: h i s 5^{+}\right)$and RPA43 $\left(\Delta r p a 43:: \mathrm{kan}^{r}\right)$ genes by homologous recombination (Longtine 
et al. 1998). The control strain, considered as the wild-type strain, was constructed by transforming the YPH500 strain with an empty pGEN vector. The level of expression of the fusion protein and of the endogenous A43 subunit was monitored by Western blot analysis (Supplemental Fig. S9). For 5S rDNA ChIP experiments, a 3HA-tag was introduced at the $\mathrm{C}$ terminus of the C160 subunit of Pol III by homologous recombination (Longtine et al. 1998).

Cells were grown in YPD medium at $30^{\circ} \mathrm{C}$. Doubling times of wild-type and CARA cells are identical (92 min; see Supplemental Fig. S1). Rapamycin (Sigma) was used at a final concentration of $0.1 \mu \mathrm{g} \cdot \mathrm{mL}^{-1}$ for Pol I purification experiments and of $0.5 \mu \mathrm{g} \cdot \mathrm{mL}^{-1}$ in other experiments.

\section{Protein purifications and analyses}

Pol I purification and Western blot analysis using polyclonal anti-Pol I and anti-A43 antibodies were performed as described (Riva et al. 1982; Peyroche et al. 2000). Treated for $30 \mathrm{~min}$ with rapamycin or not, yeast cells were harvested in mid-log phase $\left(\mathrm{OD}_{600}=1.5\right)$. Specific in vitro transcription assays using partially purified extracts (PA600) was prepared as previously described (Milkereit and Tschochner 1998). Add-back experiments were performed by addition of $200 \mathrm{ng}$ of purified Pol I to the inactive PA600 extract prepared from wild-type cells treated with rapamycin.

\section{RNAs extraction and analyses}

For total RNAs extraction, $10^{8}$ cells from mid-log phase culture were recovered by centrifugation, resuspended in $0.5 \mathrm{~mL}$ of $\mathrm{AE}$ buffer (50 mM sodium acetate buffer at $\mathrm{pH}$ 5.3, 10 mM EDTA) with $1 \%$ sodium dodecyl sulfate, and mixed with an equal volume of acid-buffered phenol (equilibrated in AE buffer). Cells were disrupted by vortexing (1 $\mathrm{min})$ at room temperature, heated at $65^{\circ} \mathrm{C}$ for $5 \mathrm{~min}$ and frozen at $-80^{\circ} \mathrm{C}$. After thawing at room temperature, samples were centrifuged $(15,000 \mathrm{~g}, 5 \mathrm{~min}$, room temperature) and $0.45 \mathrm{~mL}$ of the aqueous phase was recovered. An equal volume of phenol-dichloromethane-isoamyl alcohol (25:24:1) was added, and samples were vortexed at room temperature before being centrifuged $(15,000 \mathrm{~g}, 5 \mathrm{~min}$, room temperature). The aqueous phase was recovered, and nucleic acids were precipitated by the addition of $2.5 \mathrm{vol}$ of ethanol and $0.1 \mathrm{vol}$ of $3 \mathrm{M}$ sodium acetate $(\mathrm{pH}$ 5.3). The pellet was rinsed with $80 \%$ ethanol and dissolved in $40 \mu \mathrm{L}$ of RNase-free water treated with diethyl pyrocarbonate.

For pulse-labeling experiments, total RNAs were labeled for $20 \mathrm{~min}$ by adding $150 \mu \mathrm{Ci}$ of $\left[5,6-{ }^{3} \mathrm{H}\right]$-uracil $\left(1 \mathrm{mCi} \cdot \mathrm{mL}^{-1}\right)$ to cells in $5 \mathrm{~mL}$ of YPD medium as described (Hermann-Le Denmat et al. 1994). For each point of the time-course analysis, $3 \mu \mathrm{g}$ of total RNAs was analyzed (note that the amount of total RNAs extracted from the same number of wild-type and CARA cells decreased during the rapamycin treatment, but that for each point of the time-course analysis this amount was identical for the wild-type and the CARA cells).

For primer extension and Northern blot experiments, total RNAs extracted from $5 \times 10^{6}$ cells in mid-log phase were analyzed at each point of the time-course analysis.

Primer extension experiments were performed as described (Claypool et al. 2004) using the following oligonucleotides as primers:

35S rRNA: 5'-ACACGCTGTATAGACTAGGC-3'

RPL30: 5'-CACAGTCATGGAGAAGCATCC-3'

RPS6a: 5'-CGCCACAAACGCATCAATTAG-3'

ACT1: 5'-GATGCCAGATCTTTTCCATATCGTCCC-3'
Northern blot analyses were performed using 300-bp DNA probes generated by PCR on genomic DNA with the following oligonucleotides:

\section{5'-RPL30: 5'-AATCCCAAGAATCTATCAACC-3' \\ 3'-RPL30: 5'-AGCCAAGGTGGTCAAGATATC-3' \\ 5'-RPS6A: 5'-GAAGCCGTTGGTGACGAATTC-3' \\ 3'-RPS6A: 5'-GCTCTCTTTGGACCCAATCTC-3' \\ 5'-RPL7A: 5'-TGAACCATACGTTGCTTACGG-3' \\ 3'-RPL7A: 5'-TTACCGAAAGAACCACCTTGG-3' \\ 5'-RPL5: 5'-TCACTGGTGATGTCGTCTTAG-3' \\ 3'-RPL5: 5'-CGTCAGAAGCACCCTTTAGAG-3' \\ 5'-ACT1: 5'-CAAGACACCAAGGTATCATGG-3' \\ 3'-ACT1: 5'-TTGGATGGAAACGTAGAAGGC-3'}

PCR products were labeled with $\alpha\left[{ }^{32} \mathrm{P}\right] \mathrm{dCTP}$ by random priming (Kit Prime-it II, Stratagene).

For microarray experiments, wild-type or CARA cells were either harvested at mid-log phase $\left(\mathrm{OD}_{600}=1.0\right)$ or after an additional hour in the presence of rapamycin, or $90 \mathrm{~min}$ after the end of the log phase (during the diauxic transition). Microarray analyses were performed as described (Fauchon et al. 2002). Hybridized microarrays were scanned using a GenePix 4000A scanner (Axon Instruments, Inc.), and fluorescence ratio measurements were determined with the GenePix Pro 3.0 software (Axon Instruments, Inc.). To account for dye swap, the signalchannel and control-channel measurements for duplicated samples were reversed. A scaling factor was calculated and applied in each microarray experiment so that the median ratio value of all experiments was equal to 1 . Data analysis was performed using GeneSpring software (Silicon Genetics).

\section{Chromatin immunoprecipitation}

Chromatin immunoprecipitations were performed essentially as described (Kuras and Struhl 1999). A total of $50 \mathrm{~mL}$ of wildtype and CARA yeast cultures treated $(20 \mathrm{~min})$ or not with rapamycin were harvested in mid-log phase $\left(\mathrm{OD}_{600}=1.0\right)$ and fixed with $1 \%$ formaldehyde for $10 \mathrm{~min}$ at room temperature. Glycine was added to a final concentration of $0.4 \mathrm{M}$ and incubation continued for $5 \mathrm{~min}$. Cells were collected by centrifugation, washed once with cold $20 \mathrm{mM}$ Tris- $\mathrm{HCl}(\mathrm{pH} 8.0)$ and once with cold FA-lysis buffer (50 mM Hepes-KOH at $\mathrm{pH} 7.5,150$ $\mathrm{mM} \mathrm{NaCl}, 1 \mathrm{mM}$ EDTA, $1 \%$ Triton $\mathrm{X}-100,0.1 \%$ sodium deoxycholate, $0.1 \%$ SDS, $1 \mathrm{mM}$ PMSF), and resuspended in $500 \mu \mathrm{L}$ of cold FA-lysis buffer. A volume of $750 \mu \mathrm{L}$ of glass beads (425600 microns Glass Beads, Sigma) was added, and cells were disrupted by vortexing for $15 \mathrm{~min}$ at $4^{\circ} \mathrm{C}$. The lysate was diluted into $1.4 \mathrm{~mL}$ of cold FA-lysis buffer and the glass beads were discarded as described (Strahl-Bolsinger et al. 1997). The crosslinked chromatin was pelleted by centrifugation $(15,000 \mathrm{~g}$, $20 \mathrm{~min}, 4^{\circ} \mathrm{C}$ ), washed with $1.6 \mathrm{~mL}$ of cold FA-lysis buffer for 1 $\mathrm{h}$ at $4^{\circ} \mathrm{C}$, resuspended in $1.6 \mathrm{~mL}$ FA-lysis buffer after centrifugation, and sonicated to yield an average DNA fragment size of 400 bp (range 100-1000 bp). Finally, the samples were completed with $0.4 \mathrm{~mL}$ of cold FA-lysis buffer and clarified by centrifugation $\left(15,000 \mathrm{~g}, 30 \mathrm{~min}, 4^{\circ} \mathrm{C}\right)$.

Chromatin extract $(500 \mu \mathrm{L})$ was incubated either with monoclonal anti-CTD antibodies (8WG16, Abcam) or monoclonal anti-HA antibodies (12CA5) coupled to Dynabeads pan mouse IgG (Dynal) or polyclonal anti-A190 antibodies (Riva et al. 1982) coupled to Dynabeads sheep anti-rabbit IgG (Dynal). After $2 \mathrm{~h}$ at $\mathrm{RT}$, beads were washed once with $1.4 \mathrm{~mL}$ of FA-lysis buffer, three times with $1.4 \mathrm{~mL}$ of FA-lysis buffer with final concentration of $500 \mathrm{mM} \mathrm{NaCl}$, once with $1.4 \mathrm{~mL}$ of $10 \mathrm{mM}$ Tris- $\mathrm{HCl}$ (pH 8.0), $250 \mathrm{mM} \mathrm{LiCl,} 1 \mathrm{mM}$ EDTA, 0.5\% IGEPAL CA630 
(NP-40), 0.5\% sodium deoxycholate, and once with $1.4 \mathrm{~mL}$ of TE (10 mM Tris- $\mathrm{HCl}$ at $\mathrm{pH}$ 8.0, $1 \mathrm{mM}$ EDTA). Immunoprecipitated material was eluted from the beads by heating for $20 \mathrm{~min}$ at $65^{\circ} \mathrm{C}$ in $125 \mu \mathrm{L}$ of $25 \mathrm{mM}$ Tris- $\mathrm{HCl}(\mathrm{pH} 7.5), 5 \mathrm{mM}$ EDTA, $0.5 \%$ SDS. After recovering, the cross-link was reversed by incubating the samples with $1 \mathrm{mg} \cdot \mathrm{mL}^{-1}$ of Pronase (Roche) for 1 $\mathrm{h}$ at $37^{\circ} \mathrm{C}$ and then overnight at $65^{\circ} \mathrm{C}$. Samples were treated with $25 \mu \mathrm{g} \cdot \mathrm{mL}^{-1}$ of RNase (Eurogentec) for $1 \mathrm{~h}$ at $37^{\circ} \mathrm{C}$, and then DNA was purified using a Qiaquick spin column (Qiagen).

Immunoprecipitated and total DNA samples were quantified in triplicate by real-time PCR using the Platinum SYBR Green kit (Invitrogen) and the 7300 real-time PCR System (Applied Biosystems). Sequences of the oligonucleotide primers are available upon request. The relative IP value for a given locus is expressed as a percentage of the occupancy without rapamycin and was calculated as the ratio between the IP signal and the respective total DNA signal to correct for variation between different samples and primer pairs.

\section{Ribosomal particles analyses}

During rapamycin treatment, $10^{9}$ cells were harvested every 60 min, pelleted by centrifugation, and resuspended in $1 \mathrm{~mL}$ of dissociating buffer (without $\mathrm{MgCl}_{2}$ ) (50 mM Tris- $\mathrm{HCl}$ at $\mathrm{pH}$ 7.5, $50 \mathrm{mM} \mathrm{NaCl}, 1 \mathrm{mM}$ DTT) before addition of glass beads (0.5 mL, 425-600 microns Glass Beads, Sigma). Cells were disrupted by vortexing at $4^{\circ} \mathrm{C}(10$ times, $20 \mathrm{sec})$, and the recovered lysate was clarified by centrifugation $\left(15,000 \mathrm{~g}, 10 \mathrm{~min}, 4^{\circ} \mathrm{C}\right)$. The lysate $(0.4 \mathrm{~mL})$ was loaded onto a $5 \%-35 \%$ linear gradient of sucrose (Foiani et al. 1991) and ribosomes particles were separated by centrifugation $\left(38,000 \mathrm{rpm}, 4 \mathrm{~h}, 4^{\circ} \mathrm{C}\right)$ using a Beckman SW41 rotor. Ribosome particles were quantified by measuring the $\mathrm{A}_{254}$ of fractions collected from the bottom of the gradient.

\section{Acknowledgments}

We thank X. Gidrol (Service de Génomique Fonctionnelle, CEA/Evry, France) and Y. Frobert (Service de Pharmacologie et d'Immunologie, CEA/Saclay, France) for providing microarrays and 12CA5 antibodies, respectively. We greatly acknowledge $\mathrm{N}$. Alic for a critical reading of the manuscript, and C. Conesa and O. Harismendy for help with microarray experiments. A.L. is a recipient of a grant from the MENRT delivered by Paris-VII University.

\section{References}

Briand, J.F., Navarro, F., Gadal, O., and Thuriaux, P. 2001. Cross talk between tRNA and rRNA synthesis in Saccharomyces cerevisiae. Mol. Cell. Biol. 21: 189-195.

Brun, R.P., Ryan, K., and Sollner-Webb, B. 1994. Factor C*, the specific initiation component of the mouse RNA polymerase I holoenzyme, is inactivated early in the transcription process. Mol. Cell. Biol. 14: 5010-5021.

Clarke, E.M., Peterson, C.L., Brainard, A.V., and Riggs, D.L. 1996. Regulation of the RNA polymerase I and III transcription systems in response to growth conditions. I. Biol. Chem. 27: 22189-22195.

Claypool, J.A., French, S.L., Johzuka, K., Eliason, K., Vu, L., Dodd, J.A., Beyer, A.L., and Nomura, M. 2004. Tor pathway regulates Rrn3p-dependent recruitment of yeast RNA polymerase I to the promoter but does not participate in alteration of the number of active genes. Mol. Biol. Cell 15: 946956.

Cormack, B.P. and Struhl, K. 1992. The TATA-binding protein is required for transcription by all three nuclear RNA poly- merases in yeast cells. Cell 69: 685-696.

Crespo, J.L. and Hall, M.N. 2002. Elucidating TOR signaling and rapamycin action: Lessons from Saccharomyces cerevisiae. Microbiol. Mol. Biol. Rev. 66: 579-591.

Dernburg, A.F., Broman, K.W., Fung, J.C., Marshall, W.F., Philips, J., Agard, D.A., and Sedat, J.W. 1996. Perturbation of nuclear architecture by long-distance chromosome interactions. Cell 85: 745-759.

Desai, N., Lee, J., Upadhya, R., Chu, Y., Moir, R.D., and Willis, I.M. 2005. Two steps in Maf1-dependent repression of transcription by RNA polymerase III. J. Biol. Chem. 280: 64556462.

Deshmukh, M., Tsay, Y.F., Paulovich, A.G., and Woolford Jr., J.L. 1993. Yeast ribosomal protein L1 is required for the stability of newly synthesized 5S rRNA and the assembly of 60S ribosomal subunits. Mol. Cell. Biol. 13: 2835-2845.

Fauchon, M., Lagniel, G., Aude, J.C., Lombardia, L., Soularue, P., Petat, C., Marguerie, G., Sentenac, A., Werner, M., and Labarre, J. 2002. Sulfur sparing in the yeast proteome in response to sulfur demand. Mol. Cell 9: 713-723.

Feuerbach, F., Galy, V., Trelles-Sticken, E., Fromont-Racine, M., Jacquier, A., Gilson, E., Olivo-Marin, J.C., Scherthan, H., and Nehrbass, U. 2002. Nuclear architecture and spatial positioning help establish transcriptional states of telomeres in yeast. Nat. Cell Biol. 4: 214-221.

Foiani, M., Cigan, A.M., Paddon, C.J., Harashima, S., and Hinnebusch, A.G. 1991. GCD2, a translational repressor of the GCN4 gene, has a general function in the initiation of protein synthesis in Saccharomyces cerevisiae. Mol. Cell. Biol. 11: 3203-3216.

Gerasimova, T.I., Byrd, K., and Corces, V.G. 2000. A chromatin insulator determines the nuclear localization of DNA. Mol. Cell 6: 1025-1035.

Gourse, R.L., Gaal, T., Bartlett, M.S., Appleman, J.A., and Ross, W. 1996. rRNA transcription and growth rate-dependent regulation of ribosome synthesis in Escherichia coli. Annu. Rev. Microbiol. 50: 645-677.

Grummt, I. 2003. Life on a planet of its own: Regulation of RNA polymerase I transcription in the nucleolus. Genes \& Dev. 17: 1691-1702.

Hannan, K.M., Brandenburger, Y., Jenkins, A., Sharkey, K., Cavanaugh, A., Rothblum, L., Moss, T., Poortinga, G., McArthur, G.A., Pearson, R.B., et al. 2003. mTOR-dependent regulation of ribosomal gene transcription requires S6K1 and is mediated by phosphorylation of the carboxyterminal activation domain of the nucleolar transcription factor UBF. Mol. Cell. Biol. 23: 8862-8877.

Harismendy, O., Gendrel, C.G., Soularue, P., Gidrol, X., Sentenac, A., Werner, M., and Lefebvre, O. 2003. Genome-wide location of yeast RNA polymerase III transcription machinery. EMBO J. 22: 4738-4747.

Hermann-Le Denmat, S., Werner, M., Sentenac, A., and Thuriaux, P. 1994. Suppression of yeast RNA polymerase III mutations by FHL1, a gene coding for a fork head protein involved in rRNA processing. Mol. Cell. Biol. 14: 2905-2913.

Jorgensen, P., Rupes, I., Sharom, J.R., Schneper, L., Broach, J.R., and Tyers, M. 2004. A dynamic transcriptional network communicates growth potential to ribosome synthesis and critical cell size. Genes \& Dev. 18: 2491-2505.

Kuras, L. and Struhl, K. 1999. Binding of TBP to promoters in vivo is stimulated by activators and requires Pol II holoenzyme. Nature 399: 609-613.

Li, Y., Moir, R.D., Sethy-Coraci, I.K., Warner, J.R., and Willis, I.M. 2000. Repression of ribosome and tRNA synthesis in secretion-defective cells is signaled by a novel branch of the cell integrity pathway. Mol. Cell. Biol. 20: 3843-3851. 
Longtine, M.S., McKenzie III, A., Demarini, D.J., Shah, N.G., Wach, A., Brachat, A., Philippsen, P., and Pringle, J.R. 1998. Additional modules for versatile and economical PCR-based gene deletion and modification in Saccharomyces cerevisiae. Yeast 14: 953-961.

Marion, R.M., Regev, A., Segal, E., Barash, Y., Koller, D., Friedman, N., and O'Shea, E.K. 2004. Sfp1 is a stress- and nutrient-sensitive regulator of ribosomal protein gene expression. Proc. Natl. Acad. Sci. 101: 14315-14322.

Martin, D.E., Soulard, A., and Hall, M.N. 2004. TOR regulates ribosomal protein gene expression via PKA and the Forkhead transcription factor FHL1. Cell 119: 969-979.

Mayer, C. and Grummt, I. 2005. Cellular stress and nucleolar function. Cell Cycle 4: 1036-1038.

Mayer, C., Zhao, J., Yuan, X., and Grummt, I. 2004. mTORdependent activation of the transcription factor TIF-IA links rRNA synthesis to nutrient availability. Genes \& Dev. 18: 423-434.

Mayer, C., Bierhoff, H., and Grummt, I. 2005. The nucleolus as a stress sensor: JNK2 inactivates the transcription factor TIFIA and down-regulates rRNA synthesis. Genes \& Dev. 19: 933-941.

Milkereit, P. and Tschochner, H. 1998. A specialized form of RNA polymerase I, essential for initiation and growth-dependent regulation of rRNA synthesis, is disrupted during transcription. EMBO J. 17: 3692-3703.

Miller, G., Panov, K.I., Friedrich, J.K., Trinkle-Mulcahy, L., Lamond, A.I., and Zomerdijk, J.C. 2001. hRRN3 is essential in the SL1-mediated recruitment of RNA Polymerase I to rRNA gene promoters. EMBO I. 20: 1373-1382.

Mizuta, K. and Warner, J.R. 1994. Continued functioning of the secretory pathway is essential for ribosome synthesis. Mol. Cell. Biol. 14: 2493-2502.

Nogi, Y., Yano, R., and Nomura, M. 1991. Synthesis of large rRNAs by RNA polymerase II in mutants of Saccharomyces cerevisiae defective in RNA polymerase I. Proc. Natl. Acad. Sci. 88: 3962-3966.

Nomura, M. 2001. Ribosomal RNA genes, RNA polymerases, nucleolar structures, and synthesis of rRNA in the yeast Saccharomyces cerevisiae. Cold Spring Harb. Symp. Quant. Biol. 66: 555-565.

Oficjalska-Pham, D., Harismendy, O., Smagowicz, W.J., Gonzalez de Peredo, A., Boguta, M., Sentenac, A., and Lefebvre, O. 2006. General repression of RNA polymerase III transcription is triggered by protein phosphatase type 2A-mediated dephosphorylation of Maf1. Mol. Cell 22: 623-632.

Peyroche, G., Milkereit, P., Bischler, N., Tschochner, H., Schultz, P., Sentenac, A., Carles, C., and Riva, M. 2000. The recruitment of RNA polymerase I on rDNA is mediated by the interaction of the A43 subunit with Rrn3. EMBO J. 19: 5473-5482.

Pluta, K., Lefebvre, O., Martin, N.C., Smagowicz, W.J., Stanford, D.R., Ellis, S.R., Hopper, A.K., Sentenac, A., and Boguta, M. 2001. Maf1p, a negative effector of RNA polymerase III in Saccharomyces cerevisiae. Mol. Cell. Biol. 21: 5031-5040.

Powers, T. and Walter, P. 1999. Regulation of ribosome biogenesis by the rapamycin-sensitive TOR-signaling pathway in Saccharomyces cerevisiae. Mol. Biol. Cell 10: 987-1000.

Preiss, T., Baron-Benhamou, J., Ansorge, W., and Hentze, M.W. 2003. Homodirectional changes in transcriptome composition and mRNA translation induced by rapamycin and heat shock. Nat. Struct. Biol. 10: 1039-1047.

Reid, J.L., Iyer, V.R., Brown, P.O., and Struhl, K. 2000. Coordinate regulation of yeast ribosomal protein genes is associated with targeted recruitment of Esal histone acetylase. Mol. Cell 6: 1297-1307.
Riva, M., Buhler, J.-M., Sentenac, A., Fromageot, P., and Hawthorne, D. 1982. Natural variation in yeast RNA polymerase A. Formation of a mosaic RNA polymerase A in a meiotic segregant from an interspecific hybrid. J. Biol. Chem. 257: 4570-4577.

Roberts, D.N., Stewart, A.J., Huff, J.T., and Cairns, B.R. 2003. The RNA polymerase III transcriptome revealed by genomewide localization and activity-occupancy relationships. Proc. Natl. Acad. Sci. 100: 14695-14700.

Rohde, J.R. and Cardenas, M.E. 2003. The tor pathway regulates gene expression by linking nutrient sensing to histone acetylation. Mol. Cell. Biol. 23: 629-635.

Rudra, D. and Warner, J.R. 2004. What better measure than ribosome synthesis? Genes \& Dev. 18: 2431-2436.

Rudra, D., Zhao, Y., and Warner, J.R. 2005. Central role of Ifh 1pFhllp interaction in the synthesis of yeast ribosomal proteins. EMBO J. 24: 533-542.

Schawalder, S.B., Kabani, M., Howald, I., Choudhury, U., Werner, M., and Shore, D. 2004. Growth-regulated recruitment of the essential yeast ribosomal protein gene activator Ifh1. Nature 432: 1058-1061.

Schmelzle, T. and Hall, M.N. 2000. TOR, a central controller of cell growth. Cell 103: 253-262.

Shamji, A.F., Kuruvilla, F.G., and Schreiber, S.L. 2000. Partitioning the transcriptional program induced by rapamycin among the effectors of the Tor proteins. Curr. Biol. 10: 15741581.

Sikorski, R.S. and Hieter, P. 1989. A system of shuttle vectors and yeast host strains designed for efficient manipulation of DNA in Saccharomyces cerevisiae. Genetics 122: 19-27.

Strahl-Bolsinger, S., Hecht, A., Luo, K., and Grunstein, M. 1997. SIR2 and SIR4 interactions differ in core and extended telomeric heterochromatin in yeast. Genes \& Dev. 11: 83-93.

Tsang, C.K., Bertram, P.G., Ai, W., Drenan, R., and Zheng, X.F. 2003. Chromatin-mediated regulation of nucleolar structure and RNA Pol I localization by TOR. EMBO J. 22: 6045-6056.

Ulbrich, N., Todokoro, K., Ackerman, E.J., and Wool, I.G. 1980. Characterization of the binding of rat liver ribosomal proteins L6, L7, and L19 to $5 \mathrm{~S}$ ribosomal ribonucleic acid. J. Biol. Chem. 255: 7712-7715.

Upadhya, R., Lee, J., and Willis, I.M. 2002. Maf1 is an essential mediator of diverse signals that repress RNA polymerase III transcription. Mol. Cell 10: 1489-1494.

Wade, J.T., Hall, D.B., and Struhl, K. 2004. The transcription factor Ifh1 is a key regulator of yeast ribosomal protein genes. Nature 432: 1054-1058.

Warner, J.R. 1999. The economics of ribosome biosynthesis in yeast. Trends Biochem. Sci. 24: 437-440.

- 2001. Nascent ribosomes. Cell 107: 133-136.

Wittekind, M., Kolb, J.M., Dodd, J., Yamagishi, M., Memet, S., Buhler, J.M., and Nomura, M. 1990. Conditional expression of RPA190, the gene encoding the largest subunit of yeast RNA polymerase I: Effects of decreased rRNA synthesis on ribosomal protein synthesis. Mol. Cell. Biol. 10: 2049-2059.

Zaragoza, D., Ghavidel, A., Heitman, J., and Schultz, M.C. 1998. Rapamycin induces the G0 program of transcriptional repression in yeast by interfering with the TOR signaling pathway. Mol. Cell. Biol. 18: 4463-4470. 


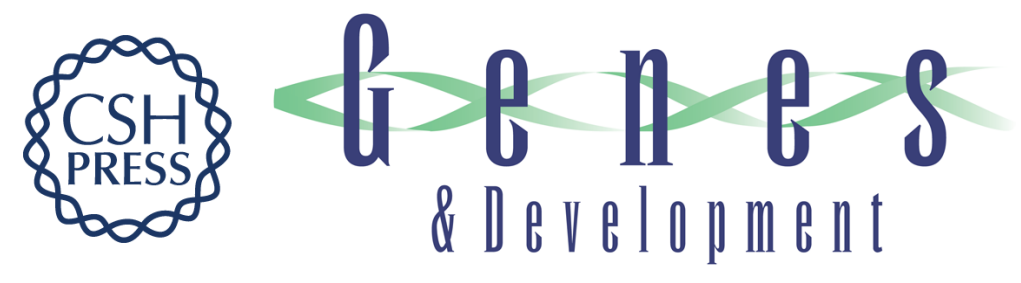

\title{
The transcriptional activity of RNA polymerase I is a key determinant for the level of all ribosome components
}

\author{
Arnaud Laferté, Emmanuel Favry, André Sentenac, et al.
}

Genes Dev. 2006, 20:

Access the most recent version at doi:10.1101/gad.386106

\section{Supplemental http://genesdev.cshlp.org/content/suppl/2006/07/14/20.15.2030.DC1 \\ Material}

Related Content

Does Pol I talk to Pol II? Coordination of RNA polymerases in ribosome biogenesis Annemieke A. Michels and Nouria Hernandez

Genes Dev. UNKNOWN , 2006 20: 1982-1985

References This article cites 56 articles, 34 of which can be accessed free at:

http://genesdev.cshlp.org/content/20/15/2030.full.html\#ref-list-1

Articles cited in:

http://genesdev.cshlp.org/content/20/15/2030.full.html\#related-urls

\section{License}

Email Alerting

Service

Receive free email alerts when new articles cite this article - sign up in the box at the top right corner of the article or click here.

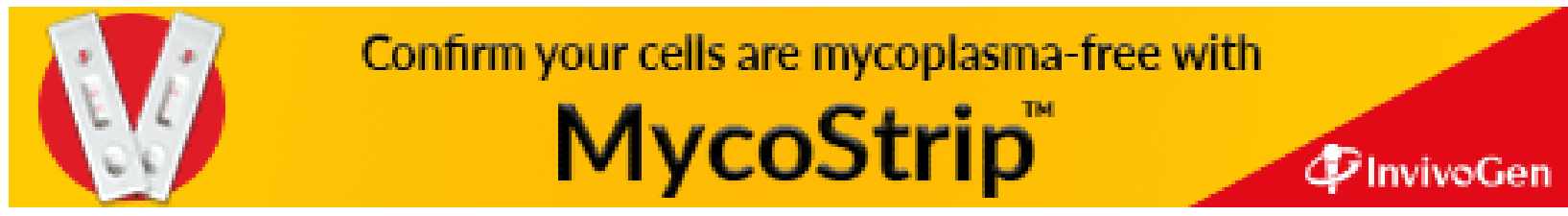

